\title{
Sentiment Analysis for Troll Detection on Weibo
}

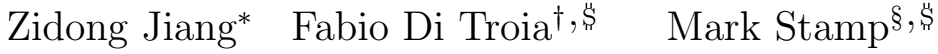

\begin{abstract}
The impact of social media on the modern world is difficult to overstate. Virtually all companies and public figures have social media accounts on popular platforms such as Twitter and Facebook. In China, the micro-blogging service provider, Sina Weibo, is the most popular such service. To influence public opinion, Weibo trolls - the so called Water Army - can be hired to post deceptive comments. In this paper, we focus on troll detection via sentiment analysis and other user activity data on the Sina Weibo platform. We implement techniques for Chinese sentence segmentation, word embedding, and sentiment score calculation. In recent years, troll detection and sentiment analysis have been studied, but we are not aware of previous research that considers troll detection based on sentiment analysis. We employ the resulting techniques to develop and test a sentiment analysis approach for troll detection, based on a variety of machine learning strategies. Experimental results are generated and analyzed. A Chrome extension is presented that implements our proposed technique, which enables real-time troll detection when a user browses Sina Weibo.
\end{abstract}

\section{Introduction}

Social media plays a significant role in the ongoing development of the Internet, as people tend to acquire more information from social media than other platforms. Deceptive comments created by trolls present a challenging problem in social media applications. Trolls can be hired to publish misleading comments in an effort to affect public opinion related to events or people, or even to negatively influence the economy of a country.

Sina Weibo is a widely used micro-blogging social media platform in China. A majority of Weibo posts are written in Chinese and, like Twitter, most posts published on Weibo are short-until recently, there was a 140 character limit. With the number of daily active users in excess of 200 million (as of 2019), Weibo is one of the largest social media platforms in China.

Weibo is based on weak relationships, in the sense that a user can share content that is visible to all of the user base. Therefore, many celebrities, businesses, and Internet influencers all over the world register as Weibo users in an effort to expand their exposure to the Chinese public. Weibo has become a platform where government and businesses can communicate more efficiently with the general public.

The Chinese Water Army refers to a group of people who can be hired to post deceptive comments on Weibo. Such troll activity is difficult to detect, due in part to the unsegmented characteristic of the Chinese sentences. In some cases, Chinese sentences can be segmented in different ways to yield different meanings.

Recent research has shown that hidden Markov models (HMM) are effective for sentiment analysis of English text [37]. Chinese word segmentation can also be accomplished using HMMs [4, 21, 32]. In this research, we use HMMs, Word2Vec and other learning techniques to perform word segmentation and sentiment analysis on Sina Weibo "tweets" for the purpose of detecting potential troll activity. We use Word2Vec and HMMs for Chinese text segmentation, we employ HMMs and naïve Bayes for sentiment analysis, and we use XGBoost and support vector machines (SVM) for troll detection.

We have generated a large training dataset by crawling the Sina Weibo and Tencent Weibo platforms. Using an HMM-based Chinese sentence segmentation model comparable to that in [32], we pre-process each post into a list of words. Then, following the approach in [10], we construct a Word2Vec similarity scoring matrix based on the word list that we have generated. A baseline of sentiment is determined from the corpus that we have collected.

*zidong.jiang@sjsu.edu

${ }^{\dagger}$ fabio.ditroia@sjsu.edu

§mark.stamp@sjsu.edu

\$D Department of Computer Science, San Jose State University, San Jose, California 
For sentiment analysis, we use a Word2Vec based technique to calculate sentiment scores. We use extracted features from Weibo comments as observations to train HMM models for each emotion, and we use the trained models to determine the emotions of each comment. We use an XGBoost model to aggregate sentiment analysis results with user activity data to build the troll detection model. As a point of comparison, we experiment with an approach based on support vector machines.

Finally, we present a Chrome extension that we have developed. This Chrome extension implements our troll detection model, and it enables us to detect potential troll activity on Weibo in real-time.

The remainder of this paper is organized as follows. In Section 2 we discuss relevant background topics. Section 3 contains an overview of selected previous work. In Section 4, we consider data sources and data collection methods. In Section 5, we provide implementation details and includes experimental results. Lastly, in Section 6 we give a summary of our work, including a brief discussion of possible directions for future development.

\section{Background}

In this section, we discuss several relevant background topics. First, to motivate this research, we discuss trolls in the context of social media. Then we introduce machine learning models that are used in this research. We conclude this section with a brief discussion of the evaluation metric that we employ.

\section{$2.1 \quad$ Trolls}

Troll users publish misleading, offensive or trivial following-up content in online communities. The content of a troll posting generally falls into one of several categories. It may consist of an apparently foolish contradiction of common knowledge, a deliberately offensive insult to the readers of a newsgroup or mailing list, or a broad request for trivial follow-up postings. The result of such posting is frequently a flood of angry responses. In some cases, the follow-up messages posted in response to a troll can constitute a large fraction of the contents of a newsgroup or mailing list on a particular topic over an extended period of time. These messages may be transmitted around the world to vast numbers of computers, wasting network resources and costing resources. Troll threads frustrate people who are trying to carry on substantive discussions [12].

Organized troll activity on the Sina Weibo platform was first detected in 2013. This initial group of troll users consisted of about 20,000 individuals in 50 ICQ chat groups associated with a person nicknamed "Daxia." Subsequently, troll activity became an online business on the Weibo platform. Trolls can be hired by businesses to publish negative comments against their competitors or to generate anonymous good reviews or positive comments. Prior to 2015, much of the troll activity on Weibo was designed to adversely affect the reputation of businesses. After 2015, stricter controls were set on speech on the Internet in China, and Sina Weibo developed a more sophisticated infrastructure to filter such troll comments. Currently, most of the troll activity on Weibo turned is designed to promote celebrities and companies.

Troll users on the Weibo platform can be categorized by their source of content. Traditionally, trolls use automated fake accounts to post repeated messages in an effort to dominate the comments. An example of such activity is shown in Figure 1. However, the Weibo platform has recently improved their infrastructure to block these repeated messages from users, based on proxy detection, combined with message filters for repeated comments.

Recently, troll users have become more sophisticated. Some organized Weibo trolls are supervised by a management group who controls what, when, and where they reply on the Weibo platform. Specific details of the comments that each troll account publishes are made by individual troll users rather than being copied from the management group. The management group only gives out the overall emotional trend that the comments should convey. Thus, content made by troll

users are repetitive but not monotonously so. This fact makes troll detection on Weibo challenging, since troll comments are composed and published by real human users. Furthermore, in recent years, trolls are mostly hired by companies and celebrities to make positive comments towards themselves. 


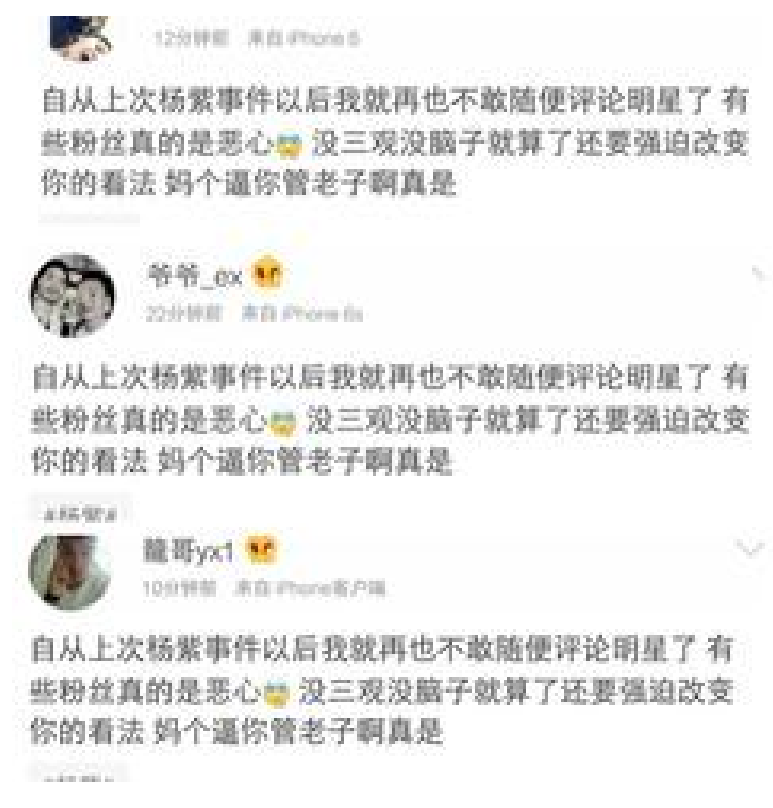

Figure 1: Weibo comments dominated by troll activity [26]

\subsection{Machine Learning Techniques}

Content-based troll detection usually utilizes natural language processing (NLP) using machine learning to analyze and categorize text. This is accomplished by constructing language processing models on comments and posts so as to label comments with a high polarity of emotion or repetitiveness. By applying sentiment analysis methods, we can filter comments with either high or low sentiment scores representing extreme positive or negative sentiment. This is accomplished by calculating word relevance, and by analyzing correlations using word embedding techniques, such as Word2Vec. We can then mark potential troll comments or pass along user information behind such comments to the next stage of a troll detection model. A key point of this research is to use sentiment analysis in troll detection.

Classifying specific comments as troll activity is challenging. Therefore, utilizing user behavioral information to discern deceptive activity is a popular trend in troll detection. Like most social media platforms, Weibo has numerous user relationship data, such as the number of followers, number that a user is following, user rank, and number of original Weibo tweets. Also, trolls commonly make attacks in a small time window following a specific tweet [11]. We can utilize this fact in combination with other user relationship information in a troll detection system. The goal of including such data in our troll detection approach is to reduce the false negatives that affect strictly content-based detection methods.

Next, we introduce the various machine learning techniques used in this research. Specifically, we discuss hidden Markov models (HMM), Word2Vec, XGBoost, and support vector machines (SVM) in some detail.

\subsubsection{Hidden Markov Models}

Hidden Markov models (HMMs) are well-known for their use in pattern prediction and for deriving hidden states from observations. An HMM (of order one) is a stochastic model representing states where each future state depends only on the current state, and not on states further in the past. By training an HMM on an observation sequence, we can obtain the probability of the transitions between each hidden state and probability distributions for the observations, based on those hidden states. A generic HMM is illustrated in Figure 2.

In Figure 2, the matrix $A$ drives the Markov process for the hidden states, while the matrix $B$ probabilistically relates the hidden states to the observations $\mathcal{O}_{i}$. The HMM notation is summarized in Table 1. 


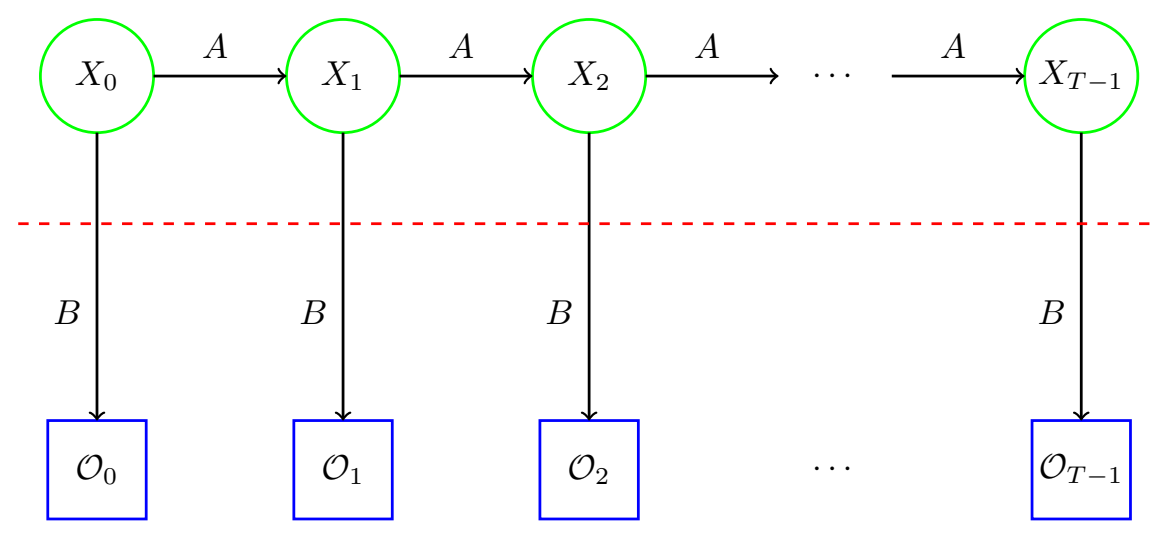

Figure 2: Hidden Markov model

Table 1: HMM notation

\begin{tabular}{cl}
\hline \hline Notation & \multicolumn{1}{c}{ Description } \\
\hline$T$ & Length of the observation sequence \\
$N$ & Number of states in the model \\
$M$ & Number of observation symbols \\
$Q$ & Distinct states of the Markov process, $q_{0}, q_{1}, \ldots, q_{N-1}$ \\
$V$ & Possible observations, assumed to be $0,1, \ldots, M-1$ \\
$A$ & State transition probabilities \\
$B$ & Observation probability matrix \\
$\pi$ & Initial state distribution \\
$\mathcal{O}$ & Observation sequence, $\mathcal{O}_{0}, \mathcal{O}_{1}, \ldots, \mathcal{O}_{T-1}$ \\
\hline \hline
\end{tabular}

Applications of HMMs are extremely diverse, but for our purposes, two relevant uses are English text analysis and speech recognition [28]. Other applications of HMMs range from classic cryptanalysis [30] to malware detection [7, 29]. In this research, we use HMMs for both Chinese word segmentation and for emotion classification.

\subsubsection{Word2Vec}

Word2Vec has recently gained considerable popularity in natural language processing (NLP) [18]. This word embedding technique is based on a shallow neural network, with the weights of the trained model serving as embedding vectors - the trained model itself serves no other purpose. These embedding vectors capture significant relationships between words in the training set. Word2Vec can also be used beyond the NLP context to model relationships between more general features or observations.

When training a Word2Vec model, we must specify the desired vector length, which we denote as $N$. Another key parameter is the window length $W$, which represents the width of a sliding window that is used to extract training samples from the data.

Certain algebraic properties hold for Word2Vec embeddings. For example, suppose that we train a state-of-the-art Word2Vec model on English text. Further, suppose that we let

$$
w_{0}=\text { "king", } w_{1}=\text { "man", } w_{2}=\text { "woman", } w_{3}=\text { "queen", }
$$

and we define $V\left(w_{i}\right)$ to be the Word2Vec embedding of word $w_{i}$. Then according to [18], the vector $V\left(w_{3}\right)$ is closest to

$$
V\left(w_{0}\right)-V\left(w_{1}\right)+V\left(w_{2}\right)
$$

where "closeness" is in terms of cosine similarity. Results such as this indicate that in the NLP context, Word2Vec embeddings capture meaningful aspects of the semantics of the language. 
In this research, we train Word2Vec models Chinese text. These models are then used for sentiment analysis of Weibo tweets.

\subsubsection{XGBoost}

Boosting is a general technique for constructing a stronger classifier from a large collection of relatively weak classifiers [6]. XGBoost typically uses decision trees as the base classifiers. To generate our models, we use the XGBoost package in Python. With this implementation, it is easy to analyze the significance of each individual feature relative to the overall model, and thus eliminate ineffective features.

\subsubsection{SVM}

In [2], support vector machine (SVM) is described as "a rare example of a methodology where geometric intuition, elegant mathematics, theoretical guarantees, and practical algorithms meet." The essential ideas behind SVMs are the following.

Separating hyperplane- We seek a hyperplane that will separate two labeled classes.

Maximize the margin - We want to find hyperplane that maximizes the "margin" between two classes, where margin is defined as the minimum distance.

Work in higher dimensional space - By shifting the problem to a higher dimensional space, there is a better chance that we can find a separation hyperplane, or separating hyperplane with a larger margin.

Kernel trick - Perhaps surprisingly, we are able to work in a higher dimensional space without paying any significant penalty with respect to computational complexity. This is a powerful "trick" and is the key reason why SVM is one of the most popular machine learning techniques available.

In this research, SVM serves as a comparison to XGBoost for troll detection. We find that XGBoost performs better on one of our datasets, while SVM is superior on another dataset.

\subsection{Evaluation Metric}

We use accuracy as the primary measure of success for all of our classification experiments. The accuracy is computed as

$$
\text { accuracy }=\frac{\mathrm{TP}+\mathrm{TN}}{\mathrm{TP}+\mathrm{TN}+\mathrm{FP}+\mathrm{FN}},
$$

where TP is number of true positive cases, TN is true negatives, FP is false positives, and FN is the number of false negatives. Accuracy can be seen to simply be the ratio of correct classifications to the total number of classifications.

Finally, we note that cross validation is used in all of our experiments. Cross validation is a popular technique that serves to smooth any bias in the data, while also maximizing the number of datapoints. Specifically, we employ 5 -fold cross validation.

\section{Related Work}

Related work in sentiment analysis includes [36], where a combination of emotional orientation and logistical regression is used to analyze Amazon.com reviews. By filtering the training dataset by text length, vocabulary complexity, correlation with the product, sentiment similarity, and transition words, the proposed model achieved $91.2 \%$ accuracy. For the problem of fake Weibo tweet detection - as opposed to the troll detection we consider in this research - an XGBoost model based on user activity achieved $93 \%$ accuracy in [17].

From our review of the literature, it appears that only [24] applies sentiment analysis to the troll detection problem. The work in [24] applies domain adaptation techniques to a recursive neural tensor network (RNTN) sentiment analysis model to detect trolls that post repetitive, destructive, or deceptive comments. This previous work achieves $78 \%$ accuracy. The results in [24] serve as a baseline for our research. 
Sentiment analysis is widely used for mining subjective information in online posts. In [14], Kim, et al., use hidden Markov models with syntactic and sentiment information for sentiment analysis of Twitter data. This differs from classic approaches that use $n$-grams and polarity lexicons, as they group words based on similar syntactic and sentiment groups (SIG), then build HMMs, where the SIGs define the hidden states. Zhao and Ohsawa [37] propose a two-dimensional HMM to analyze Amazon reviews in Japanese. For our purposes, this work illustrates an important method for converting segmented Japanese text into word vectors using Word2Vec. Feng and Durdyev [10] implemented three types of classification models (SVM, XGBoost, LSTM) for the aspect-level sentiment analysis of restaurant customer reviews in Chinese. According to the research in [10], LSTM yields better F-1 scores and accuracy, as compared to SVM and XGBoost. Further related research can be found in Liu, et al.[15], which uses a self-adaptive HMM.

Troll detection based on user characteristic data, is considered in Zhang, et al. [11]. In this paper, Weibo troll detection is based on a Bayesian model and genetic algorithm. The proposed technique includes novel features (as compared to previous work) such as the ratio of followers, average posts, and Weibo credibility, and achieves an accuracy of about $90 \%$.

Liu, Wang and Long [17] use XGBoost to detect fake Weibo posts based on features such as a user's number of posts, description, gender, followers, and reposts. The authors attain an accuracy of more than 95\%. Both [11] and [17] use data beyond Weibo post text itself, and achieve good results. This previous work serves as inspiration for some of the features considered in this paper.

The special interest group for Chinese language processing (SIGHAN) of the Association for Computational Linguistics organizes competitions for Chinese word segmentation. In the first SIGHAN bake-off event in 2003, Zhang, et al. [35] proposed a word segmentation approach using hierarchical HMMs to form a Chinese lexical analyzer, ICTCLAS. In 2005, Masayuki, et al. [1] presented three word segmentation models, including a character tagging classifier based on support vector machines (SVM) that also used maximum expropriation Markov models and conditional random fields. These models were based on previously proposed methods, with a different combination of out-of-vocabulary (OOV) extraction techniques being used.

In general, for Chinese word segmentation, character-based models perform better than wordbased models. Wang, Zong, and Su [33] highlighted that OOV techniques for word extraction performs poor for in-vocabulary (IV) words. They proposed a generative model that performs well on both OOV and IV words, and achieved good results on the popular SIGHAN datasets. Chen, Chang, and Pei [5] report the use of Gibbs sampling in combination with both word-based hierarchical process models and character-based HMMs. Their solution achieved better performance (in terms of $F_{1}$ score) than the state-of-the-art models at that time.

\section{Datasets}

We acquired data and generated additional data for the various parts of this research. We have Chinese segmentation data, sentiment analysis data, data consisting of Weibo comments, and user data corresponding to the Weibo comments data. This data is split into three datasets, namely, a Chinese segmentation dataset, a sentiment analysis dataset, and a troll detection dataset. Next, we discuss each of these three datasets.

\subsection{Chinese Segmentation Dataset}

For Chinese sentence segmentation, we acquired the dataset used in the SIGHAN 2005 Competition for Chinese sentence processing [9]. This dataset includes training, testing, and validation data. The training data consists of approximately 860,000 segmented Chinese sentences. Most of these sentences are from newspapers and published books. The test set includes about 22,000 unsegmented sentences from similar sources, while the validation set contains the segmentation of all of the sentences in the test set. Table 2 gives additional statistics for this dataset.

We consider the character-based generative model proposed in [32]. In this model, the features from the training data consist of the positions of each character in each segmented word. The beginning character in each segment is marked as $B$, any middle character or characters are marked as $M$, and the ending character is marked as $E$. On the other hand, all one-character words are marked as $S$. 
Table 2: Chinese segmentation dataset (SIGHAN 2nd Bakeoff 2005)

\begin{tabular}{c|r|r}
\hline \hline Source & Training & Testing \\
\hline Academia Sincia & 708,953 & 14,432 \\
Peking University & 19,056 & 1,944 \\
City University of Hong Kong & 53,019 & 1,492 \\
Microsoft Research Asia & 86,924 & 3,985 \\
\hline \hline
\end{tabular}

For example, consider the sample Chinese sentence in Figure 3, which includes the correct segmentation for this sentence. Table 3 gives the states corresponding to the sentence in Figure 3.

\section{马克硕士毕业于加州理工学院呀 $\downarrow$ 马克/硕士/毕业于/加州/理工/学院/呀}

Figure 3: Sample Chinese sentence segmentation

Table 3: State sequence for Chinese sentence

\begin{tabular}{c|c|c|c|c|c|c|c|c|c|c|c|c|c}
\hline \hline 马 & 克 & 硕 & 士 & 毕 & 业 & 于 & 加 & 州 & 理 & 工 & 学 & 院 & 呀 \\
\hline $\mathrm{B}$ & $\mathrm{E}$ & $\mathrm{B}$ & $\mathrm{E}$ & $\mathrm{B}$ & $\mathrm{M}$ & $\mathrm{E}$ & $\mathrm{B}$ & $\mathrm{E}$ & $\mathrm{B}$ & $\mathrm{E}$ & $\mathrm{B}$ & $\mathrm{E}$ & $\mathrm{S}$ \\
\hline \hline
\end{tabular}

\subsection{Sentiment Analysis Dataset}

For sentiment analysis, we use the sentiment training dataset from the Python SnowNLP package [34]. This particular dataset includes 16,548 sentences with positive sentiment and 18,574 with negative sentiment. The source for this dataset is Chinese online shopping, movie, and book reviews. However, this data might not accurately represents tweets and comments appearing in Weibo.

Since there is no public datasets for Weibo, we crawled about 5 million Sina Weibo posts to obtain additional data for our sentiment model. This data includes terms and slang that are commonly seen on Weibo. From this data, we created a collection of $2,325,644$ sentences with positive sentiment and 960,899 sentences with negative sentiment.

From all of our Weibo crawled data, we manually extracted 500 tweets for each of the six emotions of interest, namely, happiness, surprise, fear, anger, disgust, and sadness [3]. This data will be used to train an HMM for each emotion.

We process each comment using the Pandas package in Python to remove stop words, numbers, nonsense emoji, and single-word comments. A language detection method was implemented to detect non-Chinese comments and translate English comments into Chinese using the Translator package in Google Translate. We eliminate all comments that are in languages other than Chinese and English, which results in a negligible loss of data. In addition, we removed pure re-posts and tagging that is not relevant to our analysis.

For positive and negative sentiment analysis, we used the Word2Vec embedding method. The resulting embedding vectors enable us to calculate a word sentiment score, after segmenting a Weibo comment into a list of word. For sentiment analysis (based on six basic emotions), we use the 
features introduced in [15], which are then used to train HMMs for sentiment classification. These features are mutual information, $\chi^{2}$ distance, and term frequency inverse document frequency, which are defined as follows.

MI - Mutual information (MI) is based on correlations between two terms. In this research, MI is used to determine the relevance between words and emotions. The formula for MI representing the correlation between emotion $e$ and text $t$ is given by

$$
\mathrm{MI}(t, e)=\log \frac{P(t \mid e)}{P(e)} .
$$

CHI - We use a $\chi^{2}$ distance measure (CHI) to quantify the dependence between emotion $e$ and text $t$. The higher the CHI value, the more dependent the text $t$ is on the emotion $e$. We calculate $\mathrm{CHI}$ as

$$
\mathrm{CHI}(t, e)=\frac{N(A D-B C)^{2}}{(A+B)(C+D)(A+C)(B+D)},
$$

where $A$ is the prevalence of word $t$ in comments with emotion $e, B$ is the prevalence of word $t$ in comments with emotions other than $e, C$ is the absence of word $t$ in comments with emotion $e, D$ is the absence of word $t$ in comments with emotions other than $e$, and $N$ is the total number of comments.

TF-IDF - Term frequency inverse document frequency (TF-IDF) was originally developed to extract key words from text, for purposes such as indexing. We use TF-IDF to determine key words with respect to the various emotions under consideration. We compute the TF-IDF as

$$
\operatorname{TF}-\operatorname{IDF}(t, e)=\frac{N_{e, t}}{\sum_{k} N_{k, t}} \log \left(\frac{N}{n_{e}}+0.01\right),
$$

where $N_{e, t}$ is the number of times word $t$ appears in a comment with emotion $e, N$ is the total number of comments, and $n_{e}$ is the number of comments in which the emotion $e$ appears.

\subsection{Troll Detection Dataset}

After some initial experiments, we realized that there are limitations to the features specified in [24] and [15]. Therefore, we introduced more user information related features that we obtained by mining Weibo comment data. When crawling the Weibo data, we use the JavaScript object notation (JSON) packet returned from representational state transfer (REST) calls to the Weibo mobile site [27], which includes user-related information. Typical operations under the REST API include GET, POST, UPDATE, and DELETE. We extract the user information listed in Table 4. We also include a small dataset of 673 normal users and 75 trolls from a Kaggle data source [16].

Table 4: List of user related information from comment data

\begin{tabular}{c|c|c}
\hline \hline Field Name & Dataset & Description \\
\hline uid & UID & Unique User ID for User Account in Weibo \\
screen_name & Username & Displayed User Nickname \\
followers_count & Follower & User's follower count \\
follow_count & Following & User's following count \\
status_count & Original_post & User's original composed tweet count \\
urank & User_rank & User's rated rank by user activity in Weibo \\
verified & Verified & Whether user is verified celebrity or business \\
description & Description & User's own description in headline \\
like_count & Like_count & Like count of this comment \\
floor_number & Floor_number & Location where the comment is at \\
text & Comment & Comment content \\
\hline \hline
\end{tabular}


All user information and corresponding comments are grouped by original tweet ID and stored in CSV format. One CSV file contains all of the comments regarding one tweet, and each entry represents all of the information listed in Table 4. We selected eight tweets with a total of 31,980 comments from Sina Weibo accounts belonging primarily to business owners and celebrities. The detailed tweet information and statistics for this dataset are listed in Table 5.

Table 5: Statistics of troll detection tweets and comments crawled from Weibo

\begin{tabular}{c|c|c|r}
\hline \hline & Tweet ID & User details & Number \\
\hline 1 & 44275283 & LeEco CEO YT Jia declared bankrupt & 812 \\
2 & 44317480 & Actress Yiyan Jiang volunteered teaching in rural & 829 \\
3 & 44564209 & Yong actress Zi Yang suspected done plastic surgery & 335 \\
4 & 44718878 & Reporting fraud in singer Hong Han Foundation receiving donation & 1210 \\
5 & 44651702 & Singer Hong Han Foundation donation to Wu Han Coronavirus battle & 3379 \\
6 & 44650056 & Criticism of multiple celebrities' donation to Coronavirus battle & 814 \\
7 & 43961306 & Suspected breakup of Han Lu and Xiaotong Guan (Actor/ress) & 8371 \\
8 & 43961306 & Han Lu and Xiaotong Guan (Actor/ress) showoff their same sweatshirt & 16,230 \\
\hline \hline
\end{tabular}

We manually labeled the data for rows $1,2,3$, and 6 in Table 5 as troll or non-troll by examining the content of each comment. Combining these results with fake account data from [16], we have about 3500 comment entries for our initial training and testing data for the troll detection model. This manual labeling is extremely tedious. To accelerate this process, we created a bot based on Selenium [25] to help open each user's Weibo page based on the UID that we provide from the dataset.

To extract features from the data listed in Table 4, we use Python Pandas [20] and Numpy [19]. Some of the available features proved to be of little use, and these were dropped, as discussed below. In order to have better features for our models, we also perform some feature engineering. For example, we note whether users provide a self-description or not.

Features such as follower count, following count, and the number of original composed tweets clearly have a high significance in our analysis. However, we found that building models with quantitative numbers from these categories biases the model, due to the large differences across users. Weibo users typically only follow a fairly small number of accounts, while troll users typically follow a large number of accounts. Therefore, we dropped the follower and following count in the raw dataset and instead compute the ratio of following to follower and use this as a feature. Similarly, we introduce a feature consisting of the ratio of original posts to followers to help identify troll users, who often make a large number of posts without a commensurate increase in their follower count-we use this engineered feature in place of the composed post feature from the raw data.

When crawling Weibo, we noticed that some users frequently comment on the same tweet rather than replying to other comments under a tweet. Therefore, we select users who have more than one comment under a tweet and we count the comments made for each such user. Then we computed the median of these comment counts. Following this approach, a "frequent comment" feature is generated based on users who made more comments than the median number.

We have a total of 19 features that we use in our XGBoost model. One of the engineered features related to the sentiment score is denoted as diffOriginalSenti. This feature is the score for a comment minus the sentiment score of the original tweet. Table 6 lists the complete set of features that we obtain by combining sentiment analysis result and user information data in Table 4.

We would like to maximize our troll detection accuracy while minimizing the number of features needed. To achieve this, we perform feature analysis in order to rank the significance of features, so that we can drop features. This feature reduction process is discussed below in Section 5.5.

\section{$5 \quad$ Implementation and Results}

In this section, we give our results. First, we discuss the Weibo crawler that we have implemented. Then we consider our Chinese word segmentation results, followed by the our emotion classification 
Table 6: Features considered for troll detection model

\begin{tabular}{r|c|c|c}
\hline \hline & Feature & Description & Source \\
\hline F0 & follower & Follower count & Crawled Weibo dataset \\
F1 & following & Following count & Crawled Weibo dataset \\
F2 & original_post & Number of original tweets & Crawled Weibo dataset \\
F3 & urank & Rank by user activity in Weibo & Crawled Weibo dataset \\
F4 & verified & User certified or not & Crawled Weibo dataset \\
F5 & like_count & Like count for a comment & Crawled Weibo dataset \\
F6 & floor_number & Comment location & Crawled Weibo dataset \\
F7 & description & Self description $(1$ or 0$)$ & Engineered feature \\
F8 & freqComment & Frequent comments & Engineered feature \\
F9 & ffRatio & following divided by follower & Engineered feature \\
F10 & foRatio & original_post divided by follower & Engineered feature \\
F11 & sentiment & Comment sentiment score $(0$ to 1$)$ & Engineered feature \\
F12 & diffOriginalSenti & sentiment minus sentiment of original & Engineered feature \\
F13 & happy & Happiness score $(0$ to 1$)$ & Engineered feature \\
F14 & sad & Sadness score $(0$ to 1$)$ & Engineered feature \\
F15 & anger & Anger score $(0$ to 1$)$ & Engineered feature \\
F16 & disgust & Disgust score $(0$ to 1$)$ & Engineered feature \\
F17 & fear & Fear score $(0$ to 1$)$ & Engineered feature \\
F18 & surprise & Surprise score $(0$ to 1$)$ & Engineered feature \\
\hline \hline
\end{tabular}

technique, both of which are based on hidden Markov models. Then we consider our Word2Vec based sentiment score. and our XGBoost and SVM based troll detection results. We conclude this section with a discussion of a Chrome extension that implements this troll detection system.

\subsection{Weibo Crawler}

As mentioned above, in order to have sufficient training and testing data, we developed a crawler to obtain such data directly from the Weibo platform. Our crawler extracts posts, comments, and user information.

To extract posts, the crawler certain considers a number of tweets under specific Weibo accounts. Note that Weibo tweets are similar to Twitter tweets, in that users can retweet others users' posts to their own Weibo account. The crawler disregards retweets and only keeps original posts.

Comment crawling is used to obtain additional information related to posts. Most comments contain repetitive messages and include username and hash-tags. We remove this extraneous data with the Python Panda Dataframe function before pipelining the comments into the word segmentation stage. Also, it is very common to see bilingual comments in Weibo, where most of the text is Chinese, but some English is included. Therefore, we incorporate a language detection module extended from the Google language library, which uses naïve Bayes to filter and translate English to Chinese.

Our comment crawler works on Weibo mobile [27] data, where the tweets and comments page are slightly simplified. The crawler makes HTTP requests such as

https://m.weibo.cn/comments/hotflow?id=TWEETID\&mid=TWEETID\&max_id=

which yields JSON data from the Weibo platform containing comments related to the specified tweet. ${ }^{1}$ We parse the resulting comment data contained in the JSON packet to extract all of the raw data, including user information and comment content using the BeautifulSoup package in Python [22]. After all of the entries have been collected, they are saved into a CSV file, organized by tweet. Feature extraction and model training are based on these CSV files.

\footnotetext{
${ }^{1}$ One obstacle we encountered was a change in the Weibo mobile site at the beginning of 2020 . To avoid being blocked when crawling a large number of comments, we were forced to modify the crawler to use the "max_ID" property for the current comment page.
} 


\subsection{HMM for Chinese Segmentation}

As illustrated in Table 3, above, when segmenting a Chinese sentence, we consider four states, namely, B for begin, $\mathrm{M}$ for middle, $\mathrm{E}$ for end, and $\mathrm{S}$ for single. Thus, we train an HMM with four hidden states. The observation sequence consists of Chinese characters in the training dataset, and the hidden states correspond to B, M, E, and S. It follows that the hidden state transition matrix of the HMM is $4 \times 4$ and of the form

$$
\left[\begin{array}{cccc}
\mathrm{B} \rightarrow \mathrm{B} & \mathrm{B} \rightarrow \mathrm{E} & \mathrm{B} \rightarrow \mathrm{M} & \mathrm{B} \rightarrow \mathrm{S} \\
\mathrm{E} \rightarrow \mathrm{B} & \mathrm{E} \rightarrow \mathrm{E} & \mathrm{E} \rightarrow \mathrm{M} & \mathrm{E} \rightarrow \mathrm{S} \\
\mathrm{M} \rightarrow \mathrm{B} & \mathrm{M} \rightarrow \mathrm{E} & \mathrm{M} \rightarrow \mathrm{M} & \mathrm{M} \rightarrow \mathrm{S} \\
\mathrm{S} \rightarrow \mathrm{B} & \mathrm{S} \rightarrow \mathrm{E} & \mathrm{S} \rightarrow \mathrm{M} & \mathrm{S} \rightarrow \mathrm{S}
\end{array}\right]
$$

We implemented this HMM-based Chinese text segmentation, which is similar to that in [32]. When training, the first character of each segmented word is marked as a beginning state (B). Then characters are marked as middle states $(\mathrm{M})$, until the last character is read, which is marked as an end state (E), with any single-character words marked as such (S). The emission probability, the state transition probability, and the initial state probability are then used to update the state transition probability matrix in (1). Subsequently, we use the trained HMM to segment Weibo posts and comments line by line.

\subsection{HMM for Emotion Classification}

For each word in a tweet or comment, we can calculate a three-dimensional vector based on its MI, CHI, and TF-IDF scores, as discussed in Section 4.2, above. After calculating the feature vectors for each emotion, we obtain a mean value of each feature over all tweets labeled by each specific emotion. This mean value is used as an observation. The transition feature between states $S_{k-1}$ and $S_{k}$ is computed as

$$
P\left(S_{k}=s_{p} \mid S_{k-1}=s_{q}\right)=\left\{\begin{array}{ll}
1 & \text { if } p=q+1 \\
0 & \text { otherwise }
\end{array},\right.
$$

where the HMM states $S_{k}$ correspond to the features MI, CHI, and TF-IDF. This determines how close a feature vector in the test tweet is to those in the training set, with respect to the various emotions. The emission probability

$$
P\left(y_{k} \mid S_{k}^{e_{i}}\right)=J\left(y_{k} \mid S_{k}^{e_{i}}\right)=\frac{M_{11}}{M_{11}+M_{10}+M_{01}}
$$

can be calculated by Jaccard similarity [13], which measures the correlation between the feature vector $y_{k}$ and the state $S_{k}$, where $M_{11}$ is the total number of tweets containing feature vector $y_{k}$ and state $S_{k}$ with respect to emotion $e_{i}, M_{10}$ is the number of tweets containing only state $S_{k}$ with respect to emotion $e_{i}$, and $M_{01}$ is the number of tweets containing only feature vector $y_{k}$ with respect to emotion $e_{i}$.

Table 7 gives an example of the relationship between three consecutive words $w_{0}, w_{1}$, and $w_{2}$ in a particular test case.

We train an HMM for each of the six emotions. Then, we score a sample against each model, and assign an emotion to the tweet based on the largest probability.

\subsection{Sentiment Score Calculation}

We first construct a Word2Vec model based on the 35,124 online shopping reviews contained in the sentiment analysis dataset discussed in Section 4.2. Note that this Word2Vec model is based on segmented Chinese text. We use the GenSim package in Python [31] to train this Word2Vec model.

Next, the resulting Word2Vec embeddings are used to assign a sentiment score to segmented Chinese words, based on 3,286,543 tweets that we crawled from Weibo. Thess sentiment scores are determined using naïve Bayes. Specifically, we use naïve Bayes to compute

$$
P\left(c_{1} \mid w_{1}, \ldots, w_{n}\right)=\frac{P\left(w_{1}, \ldots, w_{n} \mid c_{1}\right) P\left(c_{1}\right)}{P\left(w_{1}, \ldots, w_{n} \mid c_{1}\right) P\left(c_{1}\right)+P\left(w_{1}, \ldots, w_{n} \mid c_{2}\right) P\left(c_{2}\right)},
$$


Table 7: Features for words $w_{0}, w_{1}$, and $w_{2}$ with respect to each emotion

\begin{tabular}{c|c|r|r|r}
\hline \hline Word & Emotion & \multicolumn{1}{c}{ MI } & \multicolumn{1}{c}{ CHI } & TD-IDF \\
\hline \multirow{6}{*}{$w_{0}$} & Happiness & 0.0012 & 0.0247 & 0.0009 \\
& Anger & 0.0012 & 0.0247 & 0.0070 \\
& Sadness & 0.0015 & 0.0100 & 0.0450 \\
& Surprise & 0.0080 & -0.0050 & 0.0220 \\
& Disgust & 0.0020 & 0.0470 & 0.0117 \\
& Fear & 0.2200 & 0.0700 & 0.0009 \\
\hline \multirow{6}{*}{$w_{1}$} & Happiness & 0.0167 & 0.0064 & 0.1045 \\
& Anger & -0.0012 & 0.0247 & 0.0009 \\
& Sadness & 0.0200 & -0.1416 & 0.0009 \\
& Surprise & 0.0012 & -0.0247 & -0.0009 \\
& Disgust & -0.0012 & 0.0247 & 0.0009 \\
& Fear & 0.0012 & 0.0247 & -0.0009 \\
\hline \multirow{6}{*}{$w_{2}$} & Happiness & 0.0012 & 0.0247 & 0.0009 \\
& Anger & 0.0012 & 0.0247 & 0.0070 \\
& Sadness & 0.0015 & 0.0100 & 0.0450 \\
& Surprise & 0.3693 & 0.0820 & -0.0119 \\
& Disgust & 0.0526 & 0.0247 & 0.0008 \\
& Fear & 0.0012 & 0.0247 & 0.0007 \\
\hline \hline
\end{tabular}

where $c_{1}$ represents the event that a word is positive and $c_{2}$ represents the event that a word is negative. Our sentiment score is computed as the product of $P\left(c_{1} \mid w_{1}, \ldots, w_{n}\right)$ and the word similarity score computed using the Word2Vec model. The resulting score can be viewed as the probability of a word in a tweet being positive, where 0 represents an extremely negative word, while 1 represents an extremely positive word. Figure 4 illustrates a sample sentiment score distribution for one of our training datasets. Note that this particular bar graph is based on the 812 comments corresponding to Weibo tweet ID 44275293, as listed in Table 5. Figure 4 shows the sentiment score frequency count distribution for all 812 comments with brackets of width 0.02 over the range of 0 to 1 .

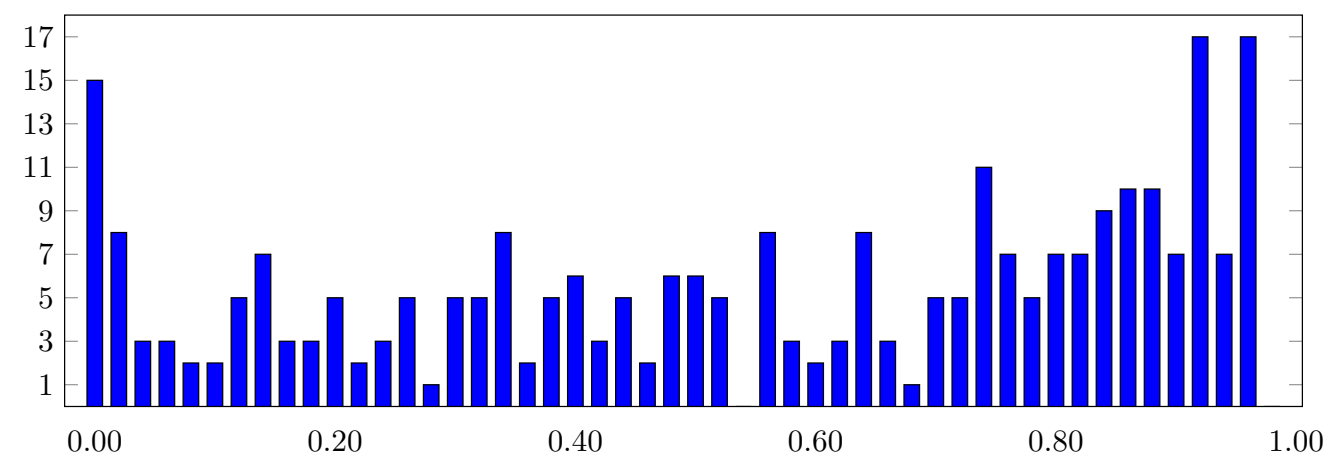

Figure 4: Sentiment score distribution for comments in Table 6, Row 1

\subsection{Troll Detection with XGBoost and SVM}

Our troll detection model is based on XGBoost. We train our XGBoost models using Python under the Jupyter Notebook environment. For these XGBoosting troll detection models, we drop 
non-quantitative features, which leaves us with the features listed in Table 8. Training an XGBoost model on all of these features, we achieve about $80 \%$ accuracy.

Table 8: Troll detection statistics crawled from Weibo

\begin{tabular}{c|c|c}
\hline \hline & Feature & Description \\
\hline F0 & follower & Follower count \\
F1 & following & Following count \\
F2 & original_post & Number of original tweets \\
F3 & ffRatio & following divided by follower \\
F4 & foRatio & original_post divided by follower \\
F5 & urank & User activity rank in Weibo \\
F6 & verified & User certified or not \\
F7 & description & User's self description (1 or 0) \\
F8 & freqComment & User comments frequently or not \\
F9 & like_count & Like count for comment \\
F10 & floor_number & Location of comment \\
F11 & sentiment & Sentiment score of the comment (0 to 1) \\
F12 & diffOriginalSenti & sentiment minus sentiment of original \\
\hline \hline
\end{tabular}

Ranking the features in this full-feature XGBoost model, we obtain the results in Figure 5. Note that F12 in Figure 5 has a weight of 0, which implies that F12 (the diffOriginalSenti feature) contributed nothing to the classification.

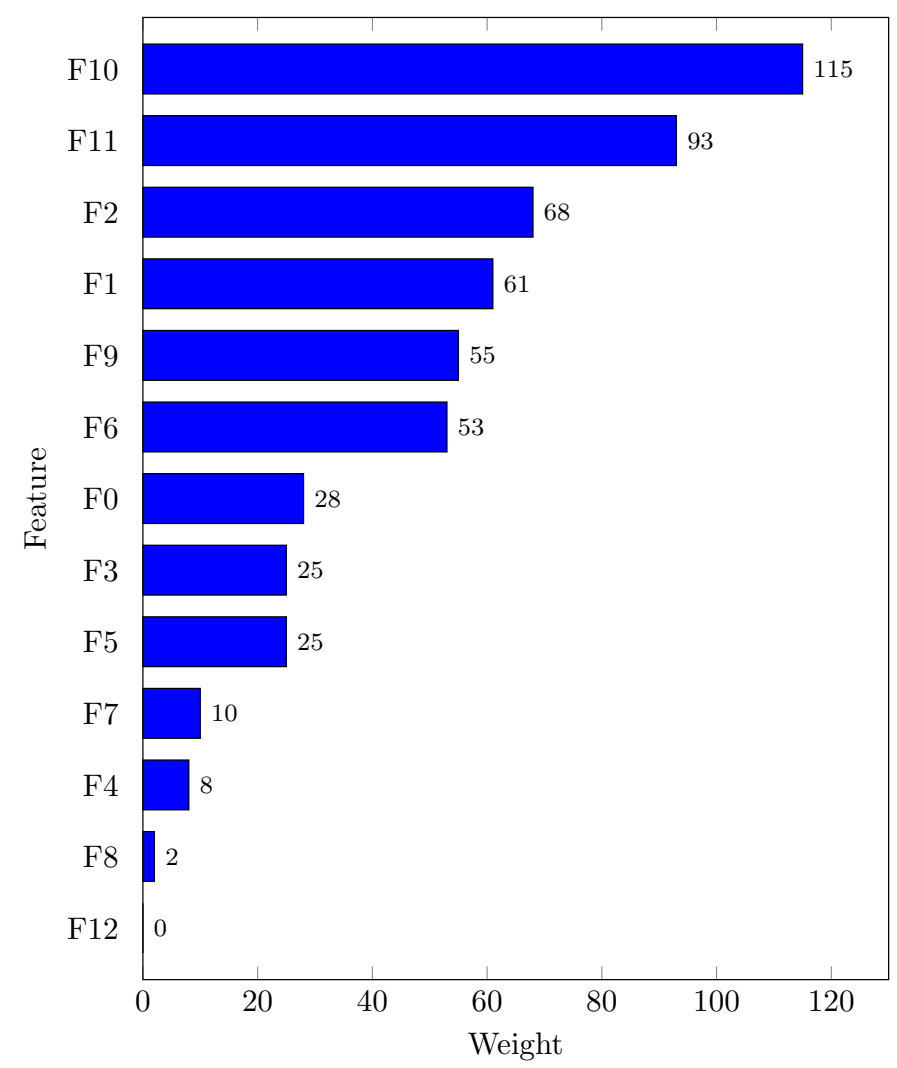

Figure 5: Initial XGBoost features ranking 
Next, we consider recursive feature elimination (RFE), where we drop the lowest ranked feature, then retrain the model. Our RFE results are given in Figure 6. We observe that the model improves when we drop the two lowest ranked features, namely, F12 and F8, but beyond that, the model will lose accuracy if we drop additional features. Hence, our optimal XGBoost model uses all of the features in Table 8, except F8 and F12. With this model, we achieve an accuracy of about $82 \%$.

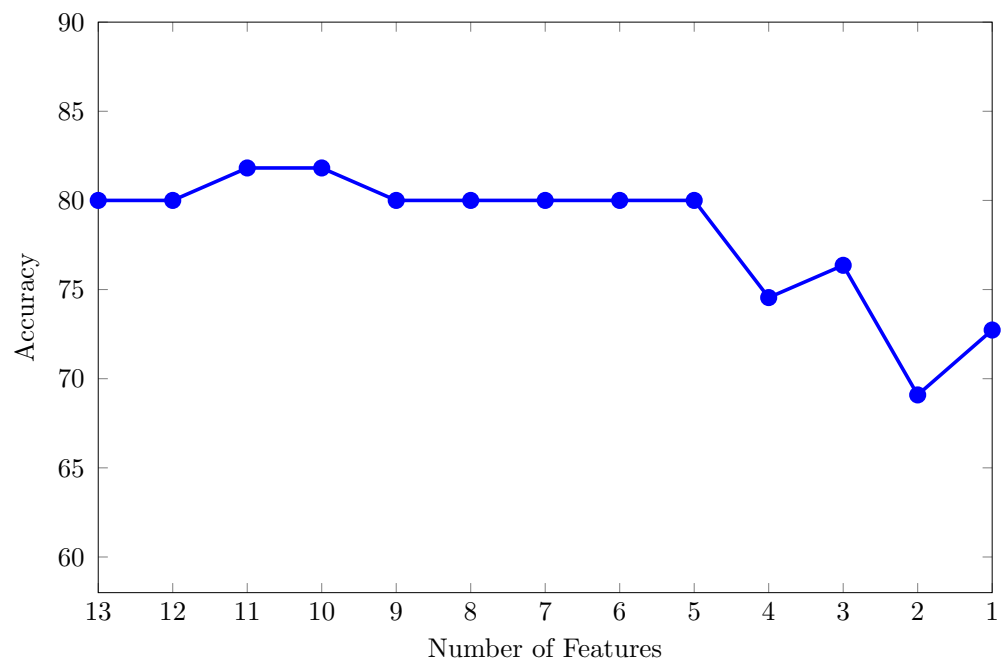

Figure 6: XGBoost model accuracy vs numbers of features

The features used and their relative importance rank are given in Figure 7. Note that these are the features in the XGBoost model.

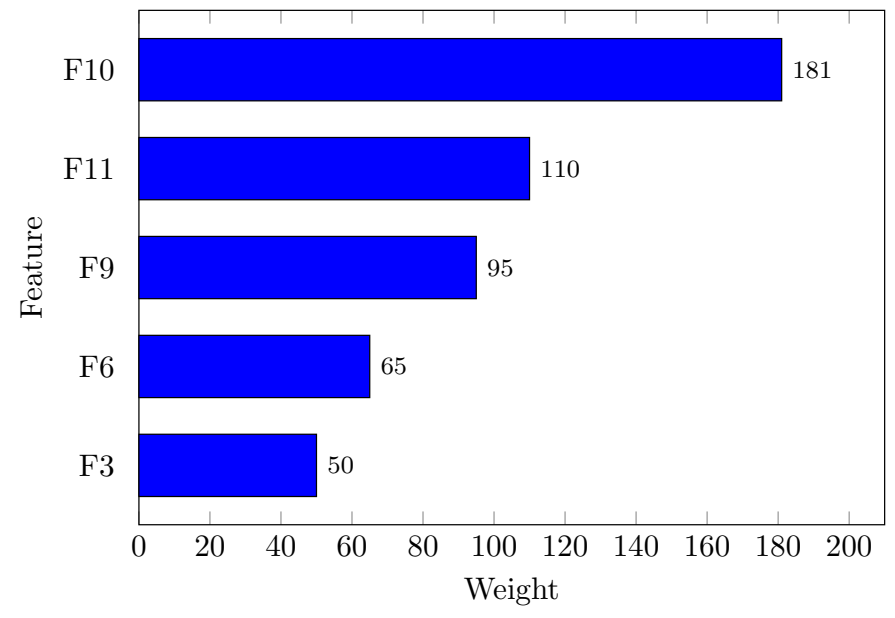

Figure 7: XGBoost features and rankings

For comparison with our XGBoost classifier, we also experiment with an SVM classifier. We utilize the Python scikit-learn package [23] to train our SVM classifier. We compare the results of these SVM classification experiments to the XGBoost experiments, based over two different datasets and various sets of features. Next, we summarize these results.

As discussed above, using XGBoost as our classification method and RFE, we achieve an accuracy of about $82 \%$. With some additional feature engineering, we were able to increase this troll detection accuracy to $83.64 \%$ on our Weibo crawled dataset, using only the three features labeled as F9, F10, F11 in Figure 7. In addition, using our SVM classifier, we achieve 87.27\% accuracy on the same dataset, based on the same three features. 
As another experiment, we compare our XGBoost and SVM models using the SnowNLP sentiment dataset for the sentiment score calculation. By using this training dataset for sentiment analysis, and with the addition of features F3 and F6, the accuracy for XGBoost is $89 \%$. However, with this same dataset and feature set, the SVM model achieves an accuracy of only $81.82 \%$. These accuracy are summarized in the form of a bar graph in Figure 8.

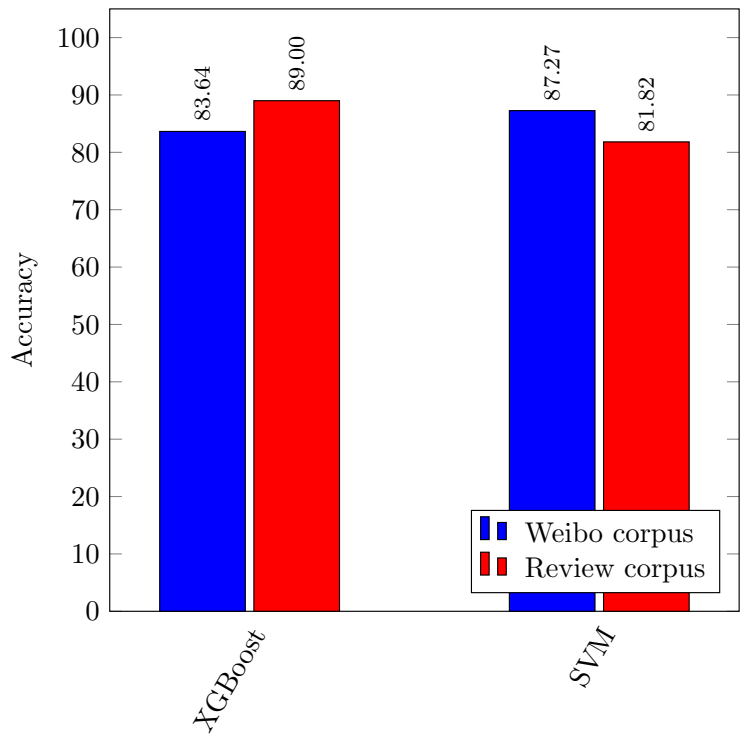

Figure 8: Comparison of XGBoost and SVM

Our experimental results show that we can achieve an accuracy as high as $89 \%$, which far exceeds the $78 \%$ accuracy obtained in the comparable previous work in [24], and matches the accuracy in the previous work [11]. A significant advantage of our approach is that it only requires a small number of easily obtained features, as compared to any previous research. This makes our troll detection technique highly efficient, and thus suitable for real-time troll detection, as validated by the Chrome extension discussed in Section 5.6, above.

\subsection{Chrome Extension for Troll Detection}

Since our troll detection mechanism is written in Python, for real-time troll detection on the Sina Weibo mobile website, we created a Chrome extension using HTML and JavaScript. In this extension, we pass the JSON packet with Weibo comment information to the back-end, which is built on the Django framework [8]. This back-end implements our troll detection model, as discussed above. The overall workflow for the plug-in is summarized as follows.

1. Run the crawler script against all the comments currently displayed in the browser under one tweet and send the packet to a server-side portal (currently running as localhost).

2. On the server-side, sort the essential user information and comment text from the returned JSON packet, as generated by the crawler.

3. On the server-side, run our sentiment analysis classifier against the comment text and acquire the text sentiment scores.

4. On the server-side, aggregate the sentiment scores and other user information, feed these into our troll detection model, and return the troll detection result to the client-side plug-in.

5. Modify the CSS style sheet for any detected troll comments by adding an orange background behind the text.

A screenshot showing this plug-in in action is given in Figure 9. Note that in this implementation, tweets and comments that have been flagged as potential troll activity are blurred. 


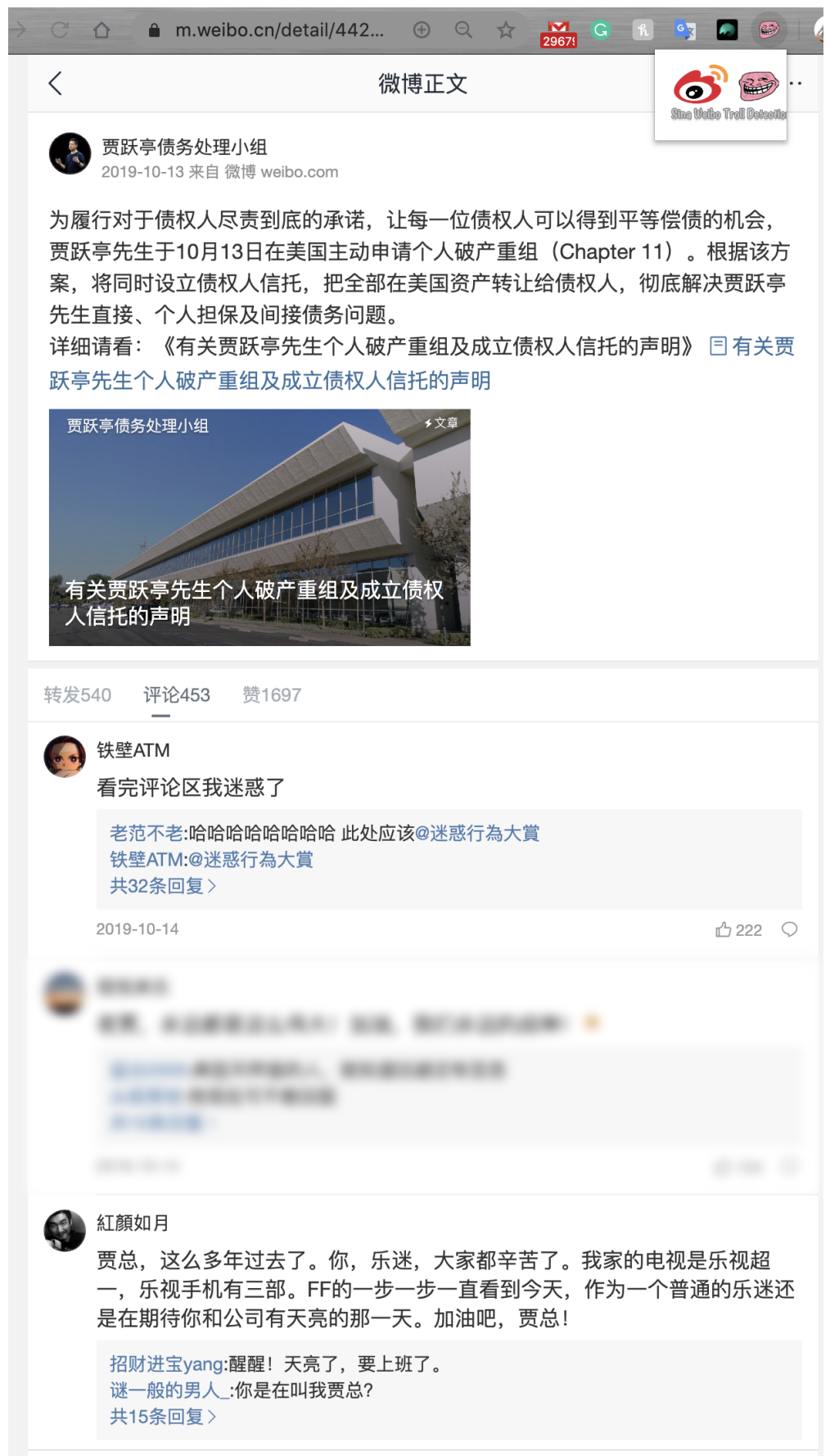

Figure 9: Chrome extension employing troll detection model

\section{Conclusion and Future Work}

The widespread use of social media enables information transfer to occur much faster than ever before. However, troll activities detract from the utility of social media. Trolls have a variety of motivations, ranging from deception to profits, and it is not likely that these motivating factors will diminish in the future. Therefore, intelligent defenses against trolls are essential.

In this research, we utilized a variety of machine learning techniques to analyze comment content and user information on the Sina Weibo platform. By conducting sentiment analysis and 
by including user data aggregation, we were able to efficiently identify troll comments on Sina Weibo with higher accuracy, as compared to previous work. We developed a Chrome extension that served to highlight the practicality of our approach.

For future work, more user data and other features can be considered. In addition, deep learning techniques that utilize sequential information, such as long short term memory (LSTM) networks, could prove useful. The Chrome extension that we have developed could be extended to support the Weibo desktop platform.

\section{References}

[1] Masayuki Asahara, Kenta Fukuoka, Ai Azuma, Chooi-Ling Goh, Yotaro Watanabe, Yuji Matsumoto, and Takashi Tsuzuki. Combination of machine learning methods for optimum Chinese word segmentation. In Proceedings of the Fourth SIGHAN Workshop on Chinese Language Processing, 2005.

[2] Kristin P. Bennett and Colin Campbell. Support vector machines: hype or hallelujah? SIGKDD Explorations, 2:1-13, 2000 .

[3] Rafael Calvo and Sunghwan Kim. Emotions in text: Dimensional and categorical models. Computational Intelligence, early view, 012012.

[4] Miaohong Chen, Baobao Chang, and Wenzhe Pei. A joint model for unsupervised chinese word segmentation. Proceedings of the 2014 Conference on Empirical Methods in Natural Language Processing, pages 854-863, 2014.

[5] Miaohong Chen, Baobao Chang, and Wenzhe Pei. A joint model for unsupervised Chinese word segmentation. In Proceedings of the 2014 Conference on Empirical Methods in Natural Language Processing, EMNLP 2014, pages 854-863. Association for Computational Linguistics, 2014.

[6] Tianqi Chen and Carlos Guestrin. XGBoost: A scalable tree boosting system. http://arxiv . org/abs/1603.02754.

[7] Dhiviya Dhanasekar, Fabio Di Troia, Katerina Potika, and Mark Stamp. Detecting encrypted and polymorphic malware using hidden Markov models. In Simon Parkinson, Andrew Crampton, and Richard Hill, editors, Guide to Vulnerability Analysis for Computer Networks and Systems: An Artificial Intelligence Approach, pages 281-299. Springer, 2018.

[8] Django Python web framework. https://www.djangoproject.com/, 2020.

[9] Thomas Emerson. The second international Chinese word segmentation bakeoff. https: //pdfs. semanticscholar.org/65e9/0d9f6754d32db464f635e7fdec672fad9ccf .pdf, 2005.

[10] Suofei Feng and Eziz Durdyev. Fine-grained sentiment analysis of restaurant customer reviews in Chinese language. http://cs229.stanford.edu/proj2018/report/195.pdf, 2018.

[11] Yingying Huang, Mengyi Zhang, Yuqing Yang, Shijie Gan, and Yanmei Zhang. The Weibo spammers' identification and detection based on Bayesian-algorithm. In 2016 2nd Workshop on Advanced Research and Technology in Industry Applications, WARTIA-16, 012016.

[12] Indiana University. What is a troll? https://kb.iu.edu/d/afhc, 2018.

[13] Paul Jaccard. Nouvelles recherches sur la distribution florale. Bulletin de la Societe Vaudoise des Sciences Naturelles, 44:223-70, 011908.

[14] N.-R Kim, Kyoungmin Kim, and Jong-Heon Lee. Sentiment analysis in microblogs using hmms with syntactic and sentimental information. International Journal of Fuzzy Logic and Intelligent Systems, 17:329-336, 122017.

[15] Li Liu, Dashi Luo, Ming Liu Liu, Jun Zhong, Ye Wei, and Letian Sun. A self-adaptive hidden Markov model for emotion classification in Chinese microblogs. Mathematical Problems in Engineering, 2015(987189), 2015.

[16] Linqing Liu, Yao Lu, Ye Luo, Renxian Zhang, Laurent Itti, and Jianwei Lu. Detecting "smart" spammers on social network: A topic model approach. In Proceedings of the NAACL Student Research Workshop, pages 45-50, San Diego, California, 2016. Association for Computational Linguistics. 
[17] Yixin Liu, Xinhua Wang, and Wen Long. Detection of false Weibo repost based on XGBoost. In IEEE/WIC/ACM International Conference on Web Intelligence, WI '19, pages 97-105. ACM, 2019.

[18] Tomas Mikolov, Kai Chen, Greg S. Corrado, and Jeffrey Dean. Efficient estimation of word representations in vector space. http://arxiv.org/abs/1301.3781, 2013.

[19] Numpy. https://numpy.org/, 2020.

[20] pandas Python data analysis library. https://pandas.pydata.org/, 2020.

[21] Haiyun Peng, Erik Cambria, and Amir Hussain. A review of sentiment analysis research in chinese language. Cognitive Computation, 8:423-435, May 2017.

[22] Leonard Richardson. BeautifulSoup Python package. https://www.crummy.com/software/ BeautifulSoup/bs4/doc/, 2020.

[23] scikit-learn Python package. https://scikit-learn.org/stable/, 2019.

[24] Chun Wei Seah, Hai Leong Chieu, Kian Ming A. Chai, Loo-Nin Teow, and Lee Wei Yeong. Troll detection by domain-adapting sentiment analysis. In 18th International Conference on Information Fusion, Fusion 2015, pages 792-799, 2015.

[25] Selenium Projects. https://www.selenium.dev/projects/, 2020.

[26] Sina-Entertainment. Weibo of Zi Yang dominated by troll. http://ent.sina.com.cn/s/m/ 2016-11-03/doc-ifxxneua4008428.shtml, 2016.

[27] Sina-Weibo. Weibo mobile site. https://m.weibo.cn/, 2009.

[28] Mark Stamp. A revealing introduction to hidden Markov model. https://www.cs.sjsu.edu/ $\sim$ stamp/RUA/HMM.pdf, 2004.

[29] Mark Stamp and S. Venkatachalam. Detecting undetectable metamorphic viruses. In Proceedings of 2011 International Conference on Security \& Management, pages 340-345, 07 2011.

[30] Rohit Vobbilisetty, Fabio Di Troia, Richard M. Low, Corrado Aaron Visaggio, and Mark Stamp. Classic cryptanalysis using hidden Markov models. Cryptologia, 41(1):1-28, 2017.

[31] Radim Řehúřek and Petr Sojka. Software framework for topic modelling with large corpora. In Proceedings of the LREC 2010 Workshop on New Challenges for NLP Frameworks, pages 45-50, 2010.

[32] Kun Wang, Chengqing Zong, and Keh-Yih Su. Which is more suitable for chinese word segmentation, the generative model or the discriminative one? Proceedings of the 23rd Pacific Asia Conference on Language, Information and Computation, pages 827-834, 2009.

[33] Kun Wang, Chengqing Zong, and Keh-Yih Su. Which is more suitable for Chinese word segmentation, the generative model or the discriminative one? In Proceedings of the 23rd Pacific Asia Conference on Language, Information and Computation, pages 827-834. City University of Hong Kong, 2009.

[34] Rui Wang. SnowNLP Python package. https://github.com/isnowfy/snownlp, 2018.

[35] Hua-Ping Zhang, Hong-Kui Yu, De-Yi Xiong, and Qun Liu. HHMM-based Chinese lexical analyzer ICTCLAS. In Proceedings of the Second SIGHAN Workshop on Chinese Language Processing, pages 184-187. Association for Computational Linguistics, July 2003.

[36] Jun Zhao and Hong Wang. Detection of fake reviews based on emotional orientation and logistic regression. Journal of CAAI Transactions on Intelligent Systems, 13:336-342, June 2016.

[37] Xiaoyi Zhao and Yukio Ohsawa. Sentiment analysis on the online reviews based on hidden Markov model. Journal of Advances in Information Technology, 9:33-38, May 2018. 